\title{
Auteur(s) et acteurs de la genèse
}

\section{Nicolas Donin et Daniel Ferrer}

\section{(2) OpenEdition}

\section{Journals}

Édition électronique

URL : http://journals.openedition.org/genesis/1440

DOI : 10.4000/genesis. 1440

ISSN : 2268-1590

\section{Éditeur :}

Presses universitaires de Paris Sorbonne (PUPS), Société internationale de génétique artistique littéraire et scientifique (SIGALES)

\section{Édition imprimée}

Date de publication : 27 novembre 2015

Pagination : 7-26

ISBN : 9791023105049

ISSN : 1167-5101

\section{Référence électronique}

Nicolas Donin et Daniel Ferrer, «Auteur(s) et acteurs de la genèse », Genesis [En ligne], 41 | 2015, mis en ligne le 10 mai 2017, consulté le 24 septembre 2020. URL : http://journals.openedition.org/genesis/ 1440 ; DOI : https://doi.org/10.4000/genesis.1440 


\section{Auteur(s) et acteurs de la genèse}

Nous avons écrit l'Anti-CEdipe à deux. Comme chacun de nous était plusieurs, ça faisait déjà beaucoup de monde. Gilles Deleuze et Felix Guattari, Rhizome 1

$Q$ uiconque se figure la fabrication d'un chef-d' œuvre de la littérature comme résultant nécessairement de l'exercice solitaire d' une souveraineté sans partage devrait jeter un coup d' œil au manuscrit de The Waste Land (fig. 1). Il se présente pour la plus grande partie comme un dactylogramme corrigé au crayon de la main de T. S. Eliot, mais on y trouve aussi des remarques timides, généralement laudatrices, inscrites en marge avec un pâle crayon par Vivien Eliot, la première épouse du poète. On est surtout frappé par de nombreuses interventions d' Ezra Pound, tantôt à l' encre, tantôt au crayon très foncé. Pound rature des passages entiers, modifie des vers, inverse des mots, constelle les marges de commentaires sarcastiques, mettant en garde contre un rythme trop ronflant, un adjectif trop banal, ou contre la trop grande ressemblance avec des poèmes antérieurs d'Eliot, avec d'autres poètes, ou, très fréquemment, avec la prose contemporaine de Joyce. En comparant ce document et la première édition de l'œuvre, souvent considérée comme le poème le plus important du XXe siècle en langue anglaise, on voit à quel point Eliot a tenu compte de ces remarques péremptoires. On a du mal à se faire à l' idée qu'un poète de cette envergure ait accepté une si lourde intervention d' une main étrangère - fût-elle celle d'un autre poète majeur. On est surpris d'apprendre que le poète se réjouissait que les manuscrits mettent en évidence cette intervention aux yeux de la postérité et encore plus peut-être de constater que sa veuve a organisé la publication en fac-similé de ces documents révélateurs. Il faut interroger cet étonnement.

Contrairement au structuralisme dont elle est issue, la critique génétique peut difficilement envisager de faire l'économie du concept d'auteur. L'intérêt pour la genèse est historiquement tributaire de la forme exacerbée qu'a revêtue, à partir du Romantisme, le culte du génie. L'étude des avant-textes participe d'une curiosité pour les mystères d'une création démiurgique conçue comme manifestation d'une disposition singulière. Cette conception a conditionné - directement ou indirectement - le recueil, la conservation et le classement des manuscrits d'écrivains. Même si les généticiens avaient voulu s'affranchir de la figure de l' "auteur » autonome et tout-puissant, ils seraient restés tributaires des matériaux qui leur étaient proposés et de l'organisation matérielle et institutionnelle sous

1. Paris, Éditions de Minuit, 1976, p. 7.

2. "In the manuscripts of The Waste Land which I am sending you, you will see evidences of [Ezra Pound's] work, and I think that this manuscript is worth preserving in its present form solely for the reason that it is the only evidence of the difference which his criticism has made to this poem », T. S. Eliot, Lettre à John Quinn, 21 septembre 1922, cité dans The Waste Land. A Facsimile and Transcript of the Original Drafts Including the Annotations of Ezra Pound, edited by Valerie Eliot, London, Faber and Faber, 1971, p. XXIV. 
laquelle ils se présentaient : l'essentiel des fonds disponibles a longtemps été des fonds d'auteur, constitués autour de la production d'un individu' ${ }^{3}$. Presque toujours, ces fonds sont assemblés à partir de matériaux filtrés par l'auteur lui-même ou par ses héritiers et même si on ne tient pas compte des cas où ont été délibérément soustraits les documents propres à affaiblir l'image du créateur en limitant fût-ce légèrement sa souveraineté exclusive sur son cuvre, cette origine ne pouvait manquer d'introduire un biais dans la collecte des matériaux.

Mais la résistance est plus profonde. On constate en fait que même quand les écrivains ont laissé subsister les documents qui démontrent une collaboration, ou quand ils se sont clairement exprimés à ce sujet, leurs témoignages sont volontiers révoqués en doute. Que nous le voulions ou non, nous avons beaucoup de mal à nous déprendre de ce que Jack Stillinger a appelé le "mythe du génie solitaire » dans un ouvrage remarquable qui est resté longtemps le seul à avoir abordé, d'un point de vue littéraire, la question des genèses à plusieurs mains ${ }^{4}$. Stillinger, spécialiste de littérature britannique du XIXe siècle, longtemps reconnu pour son travail de philologue (" textual critic ») s' efforçant de restituer la pureté des textes en extirpant les corruptions introduites par les interventions adventices, a pris ses distances avec ses propres présupposés scientifiques et mis en lumière le rôle des «amis, épouses, nègres, agents, relecteurs, censeurs, éditeurs 5 » dans le travail d'écriture des auteurs éminents qu'il avait étudiés et édités pendant des décennies sans se demander s'ils étaient les causes uniques de la totalité des textes parus sous leur nom. Contrairement à ce que l'on pourrait attendre d'une étude de la dimension collaborative de la création, l'ouvrage n'est pas consacré aux cuvres à signatures plurielles, telles que celles de Beaumont et Fletcher, Meilhac et Halévy ou Deleuze et Guattari, mais au contraire au rôle joué par des mains ignorées dans la rédaction d' œuvres célèbres. Au terme de son enquête, Stillinger conclut que l'auteur singulier pourrait bien être un mythe de civilisation et un artefact produit par le texte plutôt qu'une réalité historique.

3. L'Institut des Textes et Manuscrits modernes, qui trouve son origine dans une équipe constituée par le CNRS pour étudier les manuscrits de Heinrich Heine détenus par la Bibliothèque nationale, s'est organisé sur la base de groupes de travail consacrés chacun à l'étude de la production d'un grand écrivain : Proust, Flaubert, Zola, Valéry, Sartre, Joyce... Même si l'équipe Zola est devenue plus tard «Zola et le Naturalisme » et l'équipe Joyce, « Joyce et le Modernisme anglophone » et si d'autres équipes ont été créées sur la base de la méthodologie ou du genre littéraire, c'est seulement quand les généticiens se sont tournés vers l'architecture, le théâtre ou le cinéma qu'est apparue clairement l'impossibilité de s'en tenir à un cadre auctorial strict.

4. Jack Stillinger, Multiple Authors and the Myth of the Solitary Genius (New York, Oxford University Press, 1994). Nous pouvons nous appuyer aussi sur le travail pionnier de Guillaume Bellon et Erica Durante, coordonnateurs d'un numéro de Recto/Verso, la revue en ligne des jeunes chercheurs en critique génétique, intitulé «Écriture en collaboration » $\left(\mathrm{n}^{\circ} 3,2008\right)$. Dans l'éditorial, ils posaient ainsi le problème : «Si la signification première du mot apparaît si simple et transparente qu'elle s'est imposée à nous, il faut néanmoins démêler les sens que l'Histoire lui a surimposés. Cum-laborare : c'est dans la vérité étymologique de cette conjonction, de cette réunion autour de l'œuvre à faire que nous situons les enjeux de cette livraison. Des enjeux qui découvrent une interrogation double, quant à la figure de l'auteur et quant à l'œuvre. Ne doit-on pas, en effet, face à ces créations en collaboration, redéfinir l'instance auctoriale, dès lors que celle-ci ne peut plus être envisagée comme une individualité aux prises avec la création, mais qu'elle engage un pluriel dont la critique sait rarement quoi faire ? Et qu'est-ce qu'une ceuvre, quand elle est le fruit d'une élaboration collective, qu'elle noue les voix diverses se croisant sur la page d'écriture, la toile ou l'espace scénique ?», <www.revuerectoverso.com/spip.php?page=portfolio\&id_rubrique=39>.

5. Stillinger, op. cit., p. 182. 
La dénonciation par Stillinger de l'excessif attachement de sa discipline à la croyance en l'unicité de l'instance créatrice, vue comme un obstacle à la compréhension du caractère collaboratif des processus créateurs, incite à distinguer deux facettes de la notion d'auteur : l' "autorité " (le fait qu'une personne ou un groupe ait autorité sur un texte qui paraît sous son ou leur nom) et l' "auctorialité » (qui se rapporte aux contributions effectives d'une ou plusieurs personnes à la production d'un texte). C'est en focalisant son attention sur la seconde que Stillinger met en question la première, comme l'indique assez sa définition $d u$ "multiple authorship » (auctorialité multiple) : "production conjointe, ou composite, ou collaborative, d' euvres littéraires que nous considérons d'habitude comme rédigées par un seul auteur 6 ".

Fondée sur une telle définition, l'enquête de Stillinger l'a amené à déborder les frontières de sa spécialité d'origine et à commenter des cas d'auctorialité multiple allant de Homère au cinéma contemporain, démontrant qu'il s'agit d'un phénomène aussi banal que sous-estimé, et esquissant une typologie des formes de collaboration créatrice qui appelle continuation. Ce numéro de Genesis s'inscrit dans la même dynamique. Il souhaite mettre en évidence l'importance de l'auctorialité multiple dans tous les domaines de la création artistique. Il traverse une pluralité de configurations de collaboration, du collectif littéraire à l'interprétation musicale en passant par la cosignature de dispositifs d'exposition. Enfin, à la différence de l'essai de Stillinger et à la faveur du caractère collectif de cette publication elle-même, la question de la pluralité des mains dans les processus de création est ici formulée depuis plusieurs contextes disciplinaires différents : l'analyse génétique s'articule avec les perspectives de la sociologie, de la musicologie et de l'histoire du livre. Les pages qui suivent visent à expliciter les questions sous-jacentes à l'ensemble du numéro, afin de mettre en perspective les cas particuliers abordés par les articles et de recenser quelques-unes des conséquences génétiques de l'identification des mains multiples.

\section{Le paradigme du duo d'auteurs}

L'écriture en duo - «à quatre mains » dit-on parfois de façon trompeuse en s'inspirant de la pratique du piano - est sans doute la forme la plus connue du travail littéraire non solitaire. On l'imagine volontiers se dérouler comme cette scène récemment filmée par le documentariste Yves Jeuland, en immersion au service Politique du journal Le Monde. Deux grands reporters, Raphaëlle Bacqué et Ariane Chemin, sont dans un bureau face à leur écran d'ordinateur. Toutes deux relisent, critiquent et développent les phrases d'un article qu' elles cosigneront. Chemin, qui tient le clavier, mentionne à voix haute un propos rapporté témoignant de la relation confraternelle entre deux hommes politiques qu' explore leur article : ils s'embrassent comme le font les francs-maçons. Bacqué est intéressée par l'information mais Chemin ne veut pas paraître assigner indûment une identité francmaçonne à l'un des politiques, dans la mesure où il ne l'a pas rendue publique ; Bacqué la dissuade de passer cette notation sous silence. S'engage un travail de reformulation intensive, à l'oral et parfois à l'écran. Une formulation de prime abord satisfaisante est 
rejetée car trop sibylline pour les futurs lecteurs : "ils s'embrassent comme on le fait dans le Sud ou dans les loges ». À peine la formulation définitive arrêtée, les journalistes sont déjà plongées dans un autre passage du texte?

Dans ce moment prototypique d'écriture en duo, l'une tient la plume, l'autre oralise ; chacune apporte du matériau et produit des jugements au fur et à mesure; il y a deux points de vue critiques à satisfaire et deux sources d'encouragement; les décisions sur le texte comme sur la manière de le travailler sont prises en commun, au travers d'un dialogue qui en thématise (jusqu'à un certain point) les attendus. La caméra saisit là un témoignage exceptionnel. Imaginons un écrivain solitaire filmé en train d'écrire. On en retirerait certes des informations sur le rythme de sa production, sur les temps d'arrêt et sur l'ordre des retours en arrière, mais toute la partie délibérative nous échapperait. Le voir en train de se gratter le crâne ne nous renseignerait guère sur les enjeux ni sur les termes de ses réflexions. Dans notre exemple de collaboration dialogique, au contraire, même si les interlocutrices se comprennent à demi-mot, enjeux et arguments connaissent nécessairement une forme minimale d'explicitation. On remarque en revanche que le cour d'un tel processus de genèse collaboratif ne laisse aucune trace écrite. Certes, c'est en partie l'effet du travail sur ordinateur, qui ne fait pas apparaître les repentirs immédiats ni les différences d'écriture ou de plume. Comme le montre l'étude d'Adrien Gaillard et Julien Meyer dans le présent numéro, même une collaboration amicale et en coprésence telle celle d'Aurenche, Bost et Autant-Lara sur le scénario de Douce aura laissé des traces pour qui sait analyser leurs manuscrits, mais il est clair que bien des choses nous échappent dans une telle configuration. Les cas que nous connaissons le mieux sont ceux où les collaborateurs travaillent à distance, car leurs échanges passent par l'écrit. Ils y sont parfois obligés par l'éloignement géographique, mais il peut s'agir aussi d'une méthode délibérément choisie. François Truffaut et le scénariste Jean Gruault trouvent qu'ils se distraient mutuellement quand ils sont réunis : (presque) tout leur dialogue est donc couché sur le papier, transmis par correspondance sous forme de notes, listes de questions, versions corrigées... pour le plus grand profit des généticiens ${ }^{8}$. À l'inverse, il arrivait fréquemment dans le cinéma français que les producteurs décident de réunir tous les collaborateurs au calme dans une auberge de campagne pour faire aboutir les scénarios : ces moments de convivialité créative constituent, sinon des points aveugles, du moins des zones de faible visibilité dans l' histoire documentée de la genèse. On notera l'exemple particulier de Howard S. Becker et Robert R. Faulkner qui ont exploité leurs propres échanges de courriers électroniques pour interroger la coécriture de leur livre sur l' improvisation collective dans le jazz' .

Il serait donc trompeur de ramener tous les binômes en écriture à un modèle implicite de collaboration dans laquelle les deux contributeurs, par l'effet d'une osmose générale, verbaliseraient, écriraient et corrigeraient ensemble leur production, du projet initial aux épreuves. La lecture de la série de cas collectés par Michel Lafon et Benô̂t Peeters dans

7. Yves Jeuland, Les Gens du Monde, Paris, Folamour Productions, 2014, 82'.

8. Voir l'exposition virtuelle consacrée par la BiFi au duo Truffaut-Gruaut : «Le cinéma à quatre mains. Les films écrits par François Truffaut et Jean Gruault» (septembre 2005, consultable en ligne : <www.cinematheque.fr/ expositions-virtuelles/truffaut/reperes/analyse-detaillee/Analyse_detaillee.pdf $>$ ). Malgré ce titre, les documents présentés par le site font apparaitre que les protagonistes de cette collaboration scénaristique sont bien plus nombreux que deux.

9. Voir ci-dessous le compte rendu de ce livre par Irina Kirchberg. 
leur essai remarqué sur les «duos d'écrivains 10 » donne même à penser que l' harmonie égalitaire est une configuration minoritaire. Alexandre Dumas envoie quotidiennement des billets à son "nègre » Auguste Maquet pour lui faire produire de la copie, qu' il reformule et met au net avant de faire paraître sous son seul nom les romans ainsi produits. Eugène Labiche a écrit l'écrasante majorité de ses pièces avec l'aide de près de cinquante collaborateurs différents entre 1837 et 1878. Erckmann-Chatrian est " fondamentalement 1'association d'un auteur [Émile Erckmann] et d'un lecteur [Alexandre Chatrian]11 », et Boileau-Narcejac celle d'un scénariste et d'un écrivain ; dans les deux cas, ces duos travaillaient par correspondance, habitant dans des régions différentes. Et dans les cas rares où les coproducteurs travaillent étroitement ensemble d'un bout à l'autre du processus créateur, ce n'est pas sans une dose de pathologies physiologiques et sociales dont le couple fusionnel formé par les frères Goncourt a sans doute exploré toute la palette au fil d'un Journal conçu comme "la confession de deux vies inséparées dans le plaisir, le labeur, la peine [...] [et qui] peut être considérée comme l'expansion d'un seul moi et d'un seul je12 ».

\section{Asymétries : la gamme étendue des collaborations littéraires}

En somme, s'il est des duos égalitaires dans le travail comme dans la signature, d'autres sont fondés sur une forte différenciation des rôles et d'autres encore sont fusionnels - sans que cette diversité se reflète obligatoirement ni dans l'image de l'auteurl narrateur émanant de l'œuvre (Erckmann-Chatrian écri[ven]t volontiers "Il faut que je vous dise »), ni dans l'image publique projetée par le duo, ni dans leur exercice de l'autorité sur les auvres produites en commun. Mais les déséquilibres auctoriaux que l'enquête de Lafon et Peeters a mis en évidence de façon répétée ne sont inattendus que dans la mesure où un modèle idéalisé structure notre imaginaire, faute de familiarité avec les ressorts de la créativité collaborative. Il suffit de penser aux modes de travail mieux connus d'autres arts, plus manifestement collaboratifs, pour se rappeler qu'un duo implique avant tout une différenciation des compétences et des tâches : compositeur et librettiste d'opéra, producteur et réalisateur de cinéma, auteur et metteur en scène de théâtre peuvent concevoir ensemble une auvre, sans qu'on s'étonne de l'asymétrie des contributions respectives et sans qu'on s'émeuve de l'existence d'une hiérarchie, fixe ou mouvante. Dans le contexte littéraire, il convient aussi de prendre en compte tous les collaborateurs qui ne signent pas, depuis les nègres qui rédigent pratiquement les ouvrages clés en main pour des célébrités, jusqu'aux modestes auxiliaires qui apportent une contribution ponctuelle, comme la vérification d' un détail historique ou topographique.

Un écrivain aussi singulier et égotiste que Stendhal a requis ou proposé, tout au long de sa carrière, de multiples formes de collaboration, tantôt écrivant un livre à deux mains (Idées italiennes sur quelques tableaux célèbres 13 ), tantôt envoyant ses

10. Michel Lafon et Benoît Peeters, Nous est un autre. Enquête sur les duos d'écrivains, Paris, Flammarion, 2006. 11. Ibid., p. 146

12. Préface au Journal par Edmond de Goncourt, citée et commentée par Lafon et Peeters, op. cit., p. 23.

13. Voir dans Genesis, n 40 le compte rendu de la récente édition d'Hélène de Jacquelot et Sandra Teroni. 
manuscrits à son cousin pour qu'il les annote, tantôt aidant une amie à réviser son roman avant de s'approprier le sujet (Lucien Leuwen), tantôt se soumettant avec docilité aux suggestions de Balzac pour la réécriture de la Chartreuse de Parme, allant jusqu'à lui remettre "une Chartreuse parsemée de pages blanches qui demande vos réflexions ${ }^{14}$ " et à le prier d' "appeler un chat un chat » et de sabrer à discrétion (" effacer les lignes qui font le péché ») en portant le fer là même où ça fait le plus mal ("Dans mon amour pour le portrait de ces lieux enchanteurs je ne sens pas ce qui fait longueur 15 »). Plus fondamentalement, l'examen de ses manuscrits nous permet de dire que son écriture suppose une énonciation plurielle, feuilletée, où le destinataire tend structuralement à occuper la place de coauteur ${ }^{16}$.

On se doute que Zola, chef de file d'un mouvement littéraire, était engagé dans des collaborations multiples, même si on n'imaginait pas l'ampleur et la diversité de celles qui sont révélées par Alain Pagès ${ }^{17}$. En revanche on occulte ou on minimise souvent l'importance des partenaires chez "le solitaire de Croisset », depuis Maxime Du Camp, coauteur négligé de Par les champs et par les grèves, jusqu’à Maupassant, enquêteur pour Bouvard et Pécuchet, en passant par ces interlocuteurs fondamentaux que furent, de l'aveu même de Flaubert, Louis Bouilhet et George Sand.

Un auteur aussi individualiste et démiurgique que Joyce, qui préférait que ses ouvres restent impubliables pendant de nombreuses années plutôt que d'accepter la moindre censure, ou la moindre concession à ses éditeurs, ne travaille pourtant pas dans l'isolement. Il a le don de recruter des travailleurs bénévoles, qui effectuent pour lui de petites enquêtes à Dublin, ou qui lisent à sa demande des ouvrages, en prenant des notes, qu'il incorporera ensuite à ses textes, parfois sans aucune vérification. Il charge une dame de retranscrire les griffonnages de ses carnets, devenus illisibles pour ses yeux malades, tout en sachant qu'elle commettra nécessairement de nombreuses erreurs de déchiffrement, et il utilise ces transcriptions comme s'il s'agissait de ses propres notes, déformations comprises, qui s'intègrent au tissu de Finnegans Wake. Engagé depuis quatre ans dans l'écriture de ce livre, il suggère à Harriet Shaw Weaver, son mécène, de lui passer une commande, à la manière de celle qu'on adresse aux peintres et aux sculpteurs. Sa commanditaire lui demande alors un morceau traitant de la tombe d'un géant : Joyce s'exécute, à sa manière, et place ce morceau en ouverture du livre. Enfin, dans un moment d'épuisement et de découragement, il propose à un écrivain appelé James Stephens et né à Dublin le même jour que lui, d'achever le livre à sa place. Il suffirait, assurait-il, qu'il lui donne quelques indications, et il y parviendrait facilement... On donnerait cher pour savoir quelles auraient été ces indications, mais on comprend que Stephens ait refusé poliment!

14. Lettre du 4 avril 1841. Stendhal, Correspondance, éd. Martineau-Del Litto, Paris, Gallimard, coll. « Bibliothèque de la Pléiade », 1968, t. III, p. 433.

15. Dédicace reproduite par Alfred Hervé-Gruyer dans « Une découverte : un exemplaire dédicacé de La Chartreuse de Parme », L'Année stendhalienne 4, 2005.

16. Sur cette question et plus spécifiquement sur le projet de réécriture de la Chartreuse, voir Daniel Ferrer et Jean-Jacques Labia, «Bien vu, malentendu, mi-dit : réécritures "balzaciennes" de la Chartreuse de Parme », dans Balzac et alii, génétiques croisées. Histoires d'éditions, dir. Takayuki Kamada et Jacques Neefs, Paris, GIRBUniversité Paris Diderot, 2012 (<http://balzac.cerilac.univ-paris-diderot.fr/wa_files/FerrerLabia.pdf $>$ ).

17. Voir ci-dessous le compte rendu de l'ouvrage d'Alain Pagès, Zola et le groupe de Médan. Histoire d'un cercle littéraire, par Jean-Marc Hovasse. 
Si Joyce s'opposait farouchement à toute intervention éditoriale, il n'en est pas de même de nombreux écrivains qui n'ont d'autre choix, s'ils veulent publier, que de prendre en compte les exigences de leur maison d'édition et de laisser appliquer à leur texte un processus plus ou moins approfondi de révision, ce qu'on appelle en anglais editing. On ne saurait ranger systématiquement cette intervention sous la rubrique de l'anti-collaboration, sauf à cautionner la représentation victimaire de la relation auteur-éditeur, typiquement empreinte de la vision romantique du créateur en butte à un environnement hostile à son génie, et qui ne correspond souvent pas à la réalité.

Dans la littérature américaine, pour des raisons culturelles qu' il est impossible d'analyser ici, les choses se présentent bien autrement : les écrivains avouent sans honte la contribution de leurs éditeurs ou de leurs agents et la machine éditoriale ne se soucie pas de recouvrir à tout prix ses traces pour préserver la réputation de ses auteurs. Ainsi, on n'ignore pas qu'un roman aussi universellement célèbre que The Great Gatsby a été sensiblement modifié en collaboration avec l'éditeur. On sait que Sartoris, le livre qui a lancé Faulkner, objet d'un article célèbre de Sartre, a été remanié de fond en comble par Ben Wasson, son agent littéraire ; et on sait aussi que, même à la fin de sa carrière, après avoir obtenu le Prix Nobel, Faulkner passait des nuits entières à réviser ses manuscrits en compagnie de son éditeur, suivant docilement ses conseils. Il est possible qu'en France les choses se passent de manière moins systématique et plus artisanale, mais la pudeur est telle sur le sujet qu'il est difficile de l'affirmer. Le rôle des éditeurs de Proust dans la genèse de la Recherche du temps perdu telle que nous la connaissons a été longtemps passé sous silence et tend à être encore minimisé18. L'importance et la légitimité relatives des interventions éditoriales sont mieux admises à propos d'écrivains mineurs, ou travaillant dans des genres considérés comme mineurs, comme la littérature pour la jeunesse : on ne se sent pas obligé de détourner le regard devant les manuscrits de Jules Verne dont les marges sont remplies d'injonctions comminatoires de son éditeur-mentor Pierre-Jules Hetzel19. En Allemagne, une récente étude de sociologie historique s'est concentrée sur la profession de "lecteur 20 » du début du XXe siècle à nos jours. Le lecteur conseille la maison d'édition, accompagne l'auteur dans l'écriture, contribue à la planification des projets et à leur inscription dans une ligne éditoriale cohérente. L'analyse d'Ute Schneider s'appuie sur des cas concrets de "lecteurs » (souvent également auteurs) à l'influence avérée, tels Christian Morgenstern, Moritz Heimann, Hermann Kasack ou Friedo Lampe. Mais elle interroge aussi l'évolution, au cours du siècle, des différentes fonctions assurées par les représentants de ce métier généralement anonyme, sans cursus de formation bien identifié, et à la représentation sociale floue. L'histoire littéraire apparaît alors fortement liée à l'organisation d'un secteur économique - l'édition - dont la structure socioprofessionnelle maintient une ambiguïté constitutive entre esprit et marchandise, entre valeurs littéraires et concepts de marketing, entre auteurs et maison d'édition. Au XXIe siècle, relève Ute Schneider, le "lecteur » a été rebaptisé " product manager».

18. Nathalie Mauriac Dyer, Proust inachevé. Le dossier «Albertine disparue », Paris, Champion, 2005.

19. Philippe Scheinhardt, «Jules Verne sous la tutelle d'Hetzel. La question de la censure dans le manuscrit d'Une ville flottante », dans Catherine Viollet et Claire Bustarret (dir.), Genèse, censure, autocensure, Paris, CNRS Éditions, 2005 et «Jules Verne : un processus d'écriture sous contrainte », Genesis, n 33, 2011.

20. Ute Schneider, Die Berufsgeschichte des Lektors im literarischen Verlag, Göttingen, Wallstein, 2005. 


\section{Collectifs axiologiques}

Le type d'annotations portées par Pound sur le manuscrit de The Waste Land ne laisse pas place à l'ambiguïté : il ne s'agit pas d'une coécriture poétique, comme ce peut être le cas pour les Lyrical Ballads de Wordsworth et Coleridge, mais bien d'un travail éditorial approfondi. Pour l'essentiel, il pose des jugements : ce passage n'est pas bon et doit être supprimé, celui-ci n'est pas assez original, ce mot ou ce rythme n'est pas adéquat... Pound servait d'agent littéraire bénévole pour toute une génération de jeunes écrivains, mais tous n'avaient pas la docilité d'Eliot. Joyce, nous l'avons dit, refusait toute intervention et se rebellait notamment contre les conseils de modération de Pound. C'est qu'il avait une forte certitude de la valeur de ce qu'il écrivait (Eliot écrit qu' il avait "la vraie conviction du fanatique $21 »)$. Les collaborations qu'il sollicite visent à lui fournir des matériaux sur lesquels il exercera son appréciation en toute indépendance, ou à poursuivre un développement dans une direction qu'il aura préétablie. Un grand nombre d'écrivains (comme T. S. Eliot, ou encore, parmi ses contemporains, Virginia Woolf) ont au contraire de sérieux doutes sur la valeur, absolue ou relative, de ce qu'ils écrivent. Ils acceptent volontiers, ou même sollicitent, le jugement d'une instance plus ou moins autorisée. Une fois ce jugement émis, ils peuvent l'accepter, et modifier leur auvre dans la direction suggérée par ce jugement, ou se rebeller contre lui, quand le rapport de force, institutionnel ou affectif, leur permet de le faire. Dans les deux cas, cette confrontation axiologique (c'est-à-dire ayant trait aux valeurs et à leur hiérarchie) aura joué un rôle important.

Le jugement ainsi sollicité ou imposé serait un premier stade du contact de l'œuvre, toute pétrie des préférences idiosyncrasiques du créateur, avec le monde extérieur et les valeurs qui y ont cours 22 . Mais si l'intervention du collaborateur peut ainsi jouer le rôle d'un sas ou d'une passerelle vers la réalité, il n'est sans doute pas tout à fait exact de parler de premier contact avec les valeurs du monde extérieur. Ces valeurs, plus ou moins bien intériorisées par le processus de socialisation, sont inévitablement présentes dès le début de la création et sont souvent incarnées par des figures existantes ou fantasmées. Dans l'analyse qu' il propose de Dostö̈evski 23 , Freud fait jouer un grand rôle à un surmoi sévère qui est une intériorisation de la figure paternelle. Et sans même en remonter aux phénomènes inconscients, une branche de la psychologie contemporaine définit le « soi » comme " dialogique », donnant une place importante aux dialogues imaginaires que les humains, dès l'enfance et tout au long de la vie, entremêlent avec leurs actions, pensées, et

21. Lettre d'Eliot a John Quinn, citée dans The Waste Land. A Facsimile, op. cit., p. XX.

22. Mutatis mutandis, on peut comparer ce processus d'acclimatation de l'œuvre au travail des collaborateurs de Bruckner adaptant ses partitions au monde dans lequel elles devaient être jouées. Voir ci-dessous l'article de Pierre-Michel Menger : « la "socialisation” de l'œuvre [...] aurait commencé par anticipation, dans les décisions prises par les deux responsables du travail éditorial pour améliorer la symphonie en fonction du jugement porté sur les multiples paramètres de son interprétabilité, et en fonction de leur conviction quant au bien-fondé de leurs corrections et modifications, au vu de leur longue collaboration avec le compositeur et de leur propre expertise » (p. 67).

23. «Dostoievski et le parricide », dans Résultats, idées, problèmes, t. II, 1921-1938, Paris, PUF, 1998. 
interactions avec autrui 24 . Ces dialogues qui tissent notre existence font partie intégrante du processus de création et surtout de la phase évaluative de la genèse. Quand je me relis, je m'applique à le faire d'un oeil étranger : selon les cas, je peux adopter la perspective d'un mentor, de mes pairs ou du public (ou plus spécifiquement d'un certain public) - voire celle de l'éditeur auquel je destine mon écrit. Pour leur complaire ou pour les choquer, pour faire le beau ou pour épater le bourgeois, c'est le jugement de la figure (ou des figures) ainsi convoquée(s) qui va dicter mes corrections. Ce qu'on pourrait considérer comme la part la plus personnelle, la plus intime, du travail créateur, l'appréciation sur laquelle repose le choix, relèverait en fait d'un travail collectif, in praesentia ou in absentia. Quelle différence y a-t-il entre cette interaction avec des instances d'évaluation internalisées et les collaborations effectives, sinon celle entre le fantasme et la réalité ? Il est probable, en effet, que mon interlocuteur imaginaire, dont l'identité est définie de façon limitative par ma propre expérience, m'adresse des jugements moins inattendus et plus immédiatement compréhensibles par moi que ne le ferait un interlocuteur réel dans un dialogue réel - ne serait-ce que parce que lui-même est également composé d'instances multiples (voir en exergue la citation de Deleuze et Guattari).

A fortiori, quand les protagonistes sont plus nombreux, les dynamiques de groupe seront encore plus complexes et imprévisibles. Les groupes littéraires sont souvent moins des collectifs de production de textes que des coopératives de lecture réciproque, des laboratoires mettant au point des échelles collectives de valeur et expérimentant à l'intérieur d'un cercle restreint des normes d'acceptabilité qui peuvent être, ou non, appelées à se généraliser. Un type très particulier de collaboration axiologique se rencontre parmi les surréalistes avec la pratique des exercices de "notation scolaire », au cours desquels chacun attribuait des notes à des ouvres littéraires, des auteurs canoniques (mais aussi par exemple aux différentes parties du corps féminin). Les notes étaient soigneusement relevées et rangées dans les colonnes d'une table qui faisait apparaître des écarts considérables (Nietzsche était noté 11 par Breton et 2 par Soupault ; Mallarmé - 12 par Aragon et 15 par Éluard...). La répétition de ces expériences apparaît comme un véritable exercice collectif d'ajustement des échelles de préférence. À l'Oulipo, les normes sont théoriquement parfaitement explicitées (un texte doit répondre à une contrainte donnée) mais, à travers les documents cités dans l'article de Camille Bloomfield ici même, on perçoit une incertitude concernant d'autres valeurs (originalité, élégance, ingéniosité) qui est peut-être la véritable raison qui motive les membres à se soumettre au jugement de leurs pairs 25 .

24. Hubert J. M. Hermans et Harry J. G. Kempen, The Dialogical Self. Meaning as Movement, San Diego/London, Academic Press, 1993. Les auteurs (qui s'inspirent volontiers de la philosophie, de la littérature et de la musique pour étayer leur théorie) donnent aussi quelques exemples d'interlocuteurs imaginaires : «We find ourselves, for example, communicating with our critics, with our parents, with the photograph of someone we miss, with a figure from a movie or dream, with our consciences, with our gods, with our reflection in the mirror, with our babies, or with our pets » (p. 70).

25. Voir notamment la lettre angoissée de Luc Étienne : «plusieurs membres de l'Oulipo m'avaient dit que ce travail était l'un des meilleurs depuis longtemps. [...] je ne me ferai plus d'illusions sur l'intérêt que ma contribution peut présenter pour mes confrères » (cité par Camille Bloomfield, p. 127). 


\section{L'architecture, art collaboratif}

Ce que nous avons appelé la collaboration axiologique, volontaire ou imposée, n'est évidemment pas réservée à la littérature. Elle se rencontre dans toutes les formes d'activités créatives, mais elle n'est sans doute nulle part aussi institutionnalisée qu'en architecture $^{26}$. La confrontation des appréciations peut y prendre plusieurs aspects, mais la forme emblématique en est le concours, dans lequel un jury, nécessairement composé d'individus divers et parfois divergents, porte une appréciation, non pas sur des auvres achevées, mais sur des projets, décidant s'ils seront ou non réalisés, après les avoir éventuellement infléchis. Au-delà de cet aspect (fondamental), l'architecture est probablement l'une des formes d'activité artistique où les différentes facettes de la collaboration créative sont le plus en évidence (avec le théâtre et le cinéma, sur lesquels nous ne nous attarderons pas dans la mesure où ils sont abordés en détail par certains articles du présent numéro). Il est certes possible que la même personne décide de construire, imagine la construction et la réalise entièrement (le Facteur Cheval), mais c'est un cas exceptionnel pour les réalisations de quelque envergure. L'échelle de l'œuvre, la technicité nécessaire, les enjeux, financiers et environnementaux, enfin la durée d'exécution, qui peut aller bien au-delà de la vie d' un architecte, se conjuguent pour rendre pratiquement inévitable l'intervention de mains multiples. Nous ne nous arrêterons pas ici sur le rôle des divers corps de métier qui interagissent sur le chantier, mais nous dirons quelques mots du travail proprement architectural.

Les conflits juridiques ont presque toujours une incidence théorique et mettent souvent en lumière des questions de fond. Ainsi, à propos de la construction de la salle de la Philharmonie à Paris, un procès a récemment opposé le maître d' ouvre, Jean Nouvel, prototype $d u$ " grand architecte » contemporain, à la Ville de Paris, maître d'ouvrage. Jean Nouvel, considérant que son projet a été défiguré, réclame des modifications et refuse l'utilisation de son nom tant qu'elles n'auront pas été effectuées. Une étude approfondie des arguments échangés par les parties serait passionnante pour notre propos, mais évidemment impossible dans le cadre de cette introduction. On se contentera de citer cette déclaration de l'architecte devant le tribunal : "Rien ne se fait aux Ateliers Jean Nouvel sans moi. J'initie et je contrôle chaque chose. Je ne peux pas vous dire que j'ai tout fait, cela n'aurait pas de sens. Même les artistes contemporains aujourd' hui ne font plus leurs ouvres eux-mêmes, c'est d'une autre époque. Mais l'architecte, c'est moi. » Et cette apostrophe d'un des avocats de la partie adverse : "Notez que M. Jean Nouvel admet ne pas être l'auteur de tous les éléments du projet ${ }^{27}$."

Il est vrai qu'à notre époque, les projets importants sont généralement réalisés par de grosses agences en mettant en auvre un nombre considérable de collaborateurs, architectes salariés, dessinateurs et ingénieurs, sous la signature d'un ou deux grands noms (on

26. Voir le numéro 14 de Genesis, consacré à l'architecture, et notamment le très riche article de Pierre-Marc de Biasi, « Pour une génétique de l'architecture ». Ce numéro, qui introduisait la notion d'une génétique de l'architecture en s'appuyant tout naturellement sur le paradigme de la génétique littéraire, se plaçait pour l'essentiel dans la perspective de l'architecte comme auteur singulier, mais il mettait en évidence l'intervention d'instances multiples, visibles ou masquées, telles que celle des rédacteurs de normes.

27. « Jean Nouvel traîne son bébé en justice. Le conflit entre l'architecte et la Philharmonie de Paris se poursuit devant les tribunaux », supplément du Monde, daté du 15-16 février 2015, p. 17. 
ajoutera qu'une partie du scénario de l'auvre est déjà écrite par le commanditaire, avant même que l'agence ne se mette au travail, sous la forme du cahier des charges, dont les spécifications sont elles-mêmes la résultante d'une interaction entre diverses instances, techniques et politiques) - mais même "à une autre époque », l'architecte ne pouvait pas prétendre avoir « tout fait».

Remontons au milieu du XVII siècle et prenons le cas de Gian Lorenzo Bernini, architecte vedette s'il en fut, que Louis XIV fit venir, à grands frais et en lui rendant des honneurs princiers, pour réaliser l'aile orientale du Louvre. Mécontent des propositions des architectes français, le roi avait demandé des dessins aux principaux architectes romains et avait retenu celui du Cavalier Bernin. Nous avons la chance que tous les détails du séjour parisien du grand homme aient été pieusement relevés 28 . Nous voyons ainsi que le Bernin avait pris soin de se faire accompagner d' un assistant (" le seigneur Mathie [Mattia de Rossi], architecte travaillant sous lui 29 »), chargé d'établir les plans et de mettre au point de nombreux détails, et qui joua un rôle important dans la distribution des espaces intérieurs du palais. C'était lui qui devait assurer la surveillance du chantier, une fois son patron reparti à Rome au bout de neuf mois. Les plans et esquisses étaient régulièrement soumis à Louis XIV, et on prenait l'avis de la reine, de la maîtresse du roi, de tous les grands de la cour, dont l'opinion avait plus ou moins de poids. Même en régime despotique, les enjeux financiers sont tels que la volonté bâtisseuse, aussi ferme soit-elle, ne saurait aller sans hésitations et incertitudes. Le travail se faisait surtout en interaction constante avec Colbert, Surintendant des bâtiments du roi, qui poussait constamment l'architecte à revoir son projet, trop axé sur la façade et trop négligent de la commodité et même de la sécurité des appartements royaux. Mais, selon le Bernin, la principale difficulté était la collaboration avec ses prédécesseurs, "s'accommoder de ce qui a été fait », raccorder ses plans à l' existant, aux parties du palais déjà construites.

Ce mode de collaboration transhistorique est commun à toutes les formes d'activité artistique (on crée toujours dans le sillage d' euvres antérieures, avec elles ou contre elles), mais il prend en architecture la forme spécifique d'un dialogisme in presentia : la successivité se traduit en contiguïté spatiale. Puisque Sangallo était mort quand Michel-Ange a pris sa suite pour achever le Palais Farnèse, et que Vignola est intervenu après la mort de Michel-Ange, on ne peut pas dire que les trois grands architectes aient coopéré au sens courant du terme, mais on ne peut pas dire non plus qu'ils aient construit indépendamment un étage chacun. Michel-Ange a évidemment articulé son projet avec ce qui était déjà construit et Vignola a dû composer avec ce qu'avaient réalisé ses prédécesseurs. À plus vaste échelle, on songera à ce que la présence de la basilique Sainte-Sophie à Istanbul a pu représenter comme inspiration, comme défi et comme contrainte aux yeux des architectes ottomans : doit-on parler d'une collaboration, à un millénaire de distance, entre les concepteurs byzantins de la basilique et Mimar Sinan et ses continuateurs?

Pour revenir au Louvre et au Bernin, celui-ci n'a pas pu inscrire sa contribution dans l'espace bâti, puisque son projet a été abandonné peu après que la première pierre a été

28. Paul Fréart de Chantelou, Journal du voyage du Cavalier Bernin en France, éd. Ludovic Lalanne, Paris, Gazette des beaux-arts, 1885 .

29. Ibid., p. 28. 
posée solennellement par le roi. Ce projet demeure cependant comme un soubassement au Louvre actuel : matériellement, puisque ses successeurs ont dû faire reposer leur édifice sur les fondations qu'il avait commencé à faire poser ; mais surtout intellectuellement, puisque leurs projets ont été inévitablement établis par rapport au sien, que ce soit en opposition directe, ou parce que Colbert et Louis XIV avaient pu préciser et reformuler leurs exigences, esthétiques et matérielles, à partir des insuffisances du projet italien. Ce qu'on appelle un peu rapidement la "colonnade de Perrault ", chef-d' œuvre de l'architecture française, déjà salué comme tel par Voltaire, lui doit donc quelque chose, à côté, bien entendu, de la contribution de Claude Perrault, mais aussi de celles de Le Vau et de Le Brun, de Louis XIV et de Colbert, et de tous ceux, célèbres ou anonymes qui, pendant trois siècles, achevant pieusement le travail ou le modifiant de manière intempestive, perçant des fenêtres au XVIII siècle ou creusant des fossés sous Malraux, ont contribué à lui donner la forme que nous connaissons aujourd' hui (fig. 2).

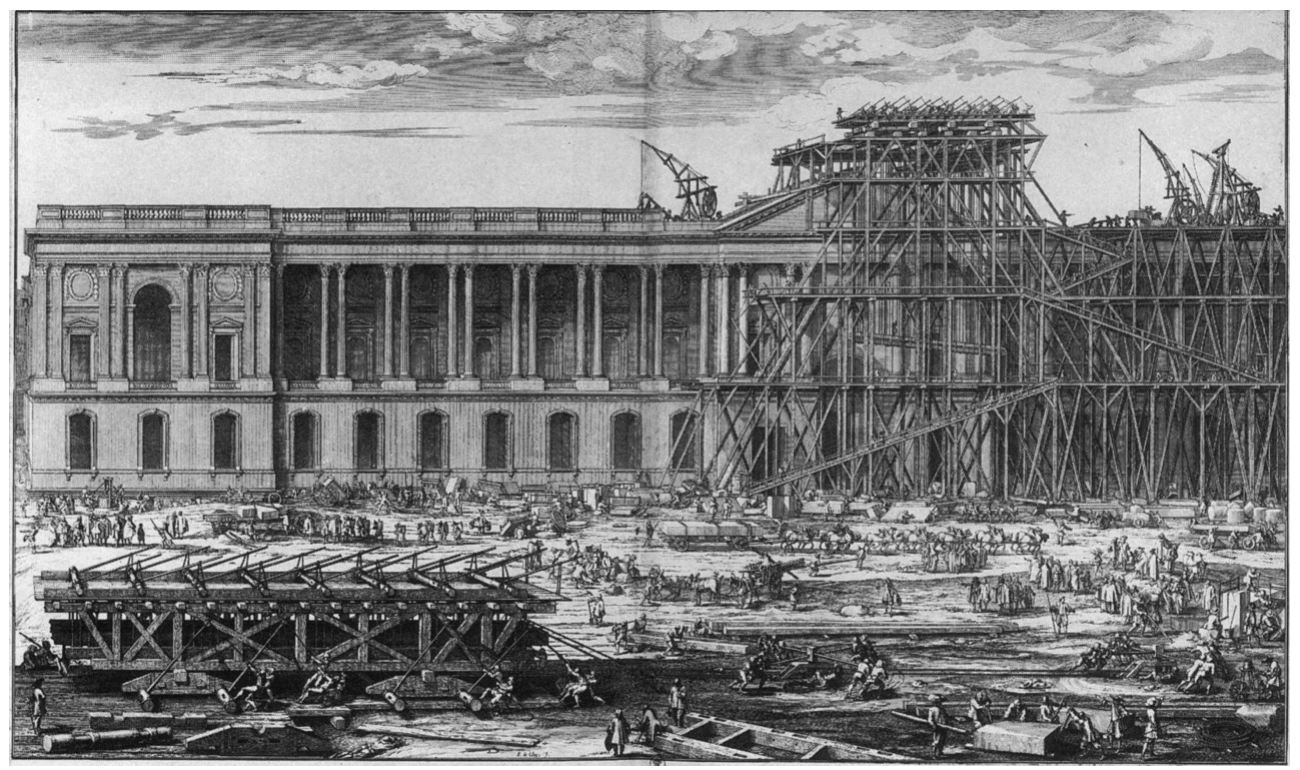

Fig. 2 : Construction de la façade orientale du Louvre ; gravure de Sébastien Leclerc

\section{La peinture : de main propre?}

La remarque de Jean Nouvel ("Même les artistes contemporains aujourd' hui ne font plus leurs ouvres eux-mêmes, c'est d'une autre époque ») étend le problème de l'auctorialité aux arts plastiques, arts essentiellement autographes dans l'acception de Nelson Goodman. Il est vrai qu'aujourd' hui beaucoup d'artistes célèbres ont des équipes nombreuses, comparables aux agences d'architectes, qui jouent un rôle primordial dans la réalisation des ceuvres. On pourrait donc y voir une rupture par rapport au modèle traditionnel de l'artiste, seul devant son chevalet pour produire une toile où chaque touche de son pinceau constitue l'équivalent d'une signature. Mais si ce modèle reste 
encore prégnant, il ne faut pas en surestimer l'universalité30. Sans même aller chercher au loin des civilisations dans lesquelles une telle conception de l'art et des artistes est tout simplement inimaginable, il suffit de reculer un peu dans le temps pour trouver des pratiques d'atelier, très diverses, qui soulèvent à différents degrés les questions de l'auctorialité et de l'autorité. On sait qu'au XVIe siècle à Venise ${ }^{31}$ ou au XVIIe à Anvers ${ }^{32}$, le nom de l'artiste, sa signature, mais aussi sa manière, pouvaient se transmettre, comme un fonds de commerce, de père en fils ou de maître à disciple. Il s'agissait d'une marque de fabrique, sinon industrielle du moins artisanale, certes issue de la pratique singulière d'un artiste reconnu, mais déclinée par plusieurs mains. Dans la mesure où l' héritier fait évoluer la marque 33 , on peut la considérer comme l'objet d'une coopération transgénérationnelle.

Mais c'est aussi à l'intérieur de chaque tableau que peut s'exercer, et que s'exerce très fréquemment, une collaboration créative. En dehors des circonstances où l'héritier termine les tableaux que le maître avait dî laisser inachevés, on retrouve régulièrement les cas suivants : le maître délègue à un collaborateur spécialisé, et éventuellement plus habile que lui dans ce domaine, l'exécution d'une partie du tableau (paysages de fond, verdures, animaux...) ; ayant exécuté le visage d'un portrait, il laisse à ses disciples le soin d'exécuter le corps et les mains ; ayant ébauché le tableau, il confie à son atelier l'essentiel de l'exécution ; pour répondre à la demande, il fait reproduire une de ses ouvres par ses collaborateurs, éventuellement avec des variantes de leur cru; l'atelier produit une ouvre sur la base d'une simple esquisse du maître ; les ouvres produites par un disciple sont retouchées de la main du maître... Dans tous ces cas de figure, la signature du maître peut figurer sur le tableau. Les recherches les plus récentes tendent à montrer, au grand dam de la tradition attributionniste de l'histoire de l'art et $d u$ connoisseurship, que pour un certain nombre d'artistes fameux on est incapable de définir un "noyau dur de l'Euvre " qui serait constitué de tableaux exécutés de la seule main du maître à partir duquel il serait possible d'identifier ceux qui sont entachés de collaborations ancillaires ${ }^{34}$.

Si l'histoire de l'art a pu progressivement penser de façon critique cette problématique de la "main », c'est peut-être aussi parce que certains artistes modernes et postmodernes en ont fait un enjeu central de leur travail. De Warhol à Koons en passant par l'art conceptuel, déléguer la réalisation de l'œuvre est devenu une pratique artistique en soi. Que ce soit pour critiquer le statut social de l'artiste ou pour le renforcer pragmatiquement par augmentation de sa productivité, la distinction entre conception et réalisation est longtemps allée de pair avec un maintien du caractère souverain de la signature. Cette dernière a fait l'objet, à son tour, d'expérimentations dont la plus radicale est peut-

30. Voir à ce sujet De l'authenticité. Une histoire des valeurs de l'art (XVIe-XXe siècle), dir. Charlotte Guichard, Paris, Publications de la Sorbonne, 2014.

31. Carlo Corsato, «Authenticité et autorialité à Venise au XVIe siècle. Sources littéraires et pratiques artistiques », dans De l'authenticité, op. cit., p. 21-44.

32. Elizabeth A. Honig, Painting and the Market in Early Modern Antwerp, New Haven, Yale University Press, 1998.

33. Voir l'exemple des Bassano évoqué dans Carlo Corsato, loc. cit.

34. Voir Art Market and Connoisseurship: A Closer Look at Paintings by Rembrandt, Rubens and Their Contemporaries, dir. Anna Tummers et Koenraad Jonckheere, Amsterdam, Amsterdam University Press, 2008. 
être celle de Philippe Thomas, fondateur d'une agence nommée "Les ready-made appartiennent à tout le monde ", dont l'activité consista à vendre des euvres dont les acquéreurs, par contrat, devenaient les auteurs. Au-delà de ce cas extrême, de nombreux artistes des années quatre-vingt et quatre-vingt-dix ont multiplié les expériences de délégation et de partage d'autorité. Pierre Joseph, qui se réfère à la figure de Thomas dans l'entretien que nous publions, est l'un d'entre eux. Sans doute n'est-ce pas un hasard si, dans le cadre de ses æeuvres récentes autour des thèmes de l'apprentissage et de l'ignorance, il a animé une sorte d'anti-« atelier » de maître où les étudiants étaient invités à dessiner de mémoire, sans aide ni support externe, les cartes de leur quartier, de la France ou de parties du corps (un échantillon du résultat est visible p. 142), aboutissant à une exposition et une publication sous son nom.

\section{Simultanéités, successivités, alternances : (co)opérer dans le temps}

Les cas de figure variés que nous venons de passer en revue montrent bien que les diverses formes de collaborations ne s'inscrivent pas de la même manière dans le temps. Il semble au premier abord nécessaire de distinguer les collaborations simultanées et les collaborations successives.

Dans la première catégorie, il y aurait les écrivains qui, comme Chemin et Bacqué, le duo de journalistes filmées devant leur écran, travaillent ensemble sur leur texte. Il y aurait aussi bien les musiciens qui improvisent de concert, comme les musiciens de jazz étudiés par Becker et Faulkner, ou encore les improvisateurs de théâtre. Mais aussi les collaborateurs d'un film réunis par leur producteur dans l'emblématique auberge de campagne.

Dans la deuxième catégorie, il y aurait avant tout les protagonistes de ce qu' Henri Gouhier appelle les arts à deux temps et Nelson Goodman les arts à deux phases. Le compositeur d'une partition musicale, l'auteur d'une pièce de théâtre, ont besoin de la collaboration, dans un deuxième temps, d'un ou plusieurs interprètes pour que leurs ceuvres connaissent leur accomplissement. Le fait qu'un virtuose puisse composer pour lui-même, qu'un comédien puisse écrire les textes de ses one man shows, n'y change pas grand-chose. Il s'agit de positions occupées par des instances structuralement déterminées. À l'inverse, il est d'usage que l'architecte supervise la construction de l'édifice dont il a tracé les plans, mais ce n'est pas toujours le cas, pour des raisons diverses : parce que le marché de la surveillance du chantier a été remporté par un moins-disant; parce qu'il a vendu ses plans à un promoteur qui les reproduira à de nombreux exemplaires au fil des années et à travers le territoire; parce que, comme le Bernin, il veut rentrer en Italie avant l'hiver; ou tout simplement parce qu' il est mort.

Mais cette dernière cause d'interruption peut tout aussi bien intervenir à l'intérieur d'une même phase : on songe à Eybler et Süssmayr achevant la partition du Requiem "de Mozart» avec la piété de disciples accoutumés à travailler à ses côtés, mais aussi à Friedrich Cerha complétant l'opéra Lulu " de Berg », soixante-cinq ans après la mort de son concepteur. La même chose se produit, nous l'avons vu, dans les arts à phase unique, comme la peinture. Les exemples ne manquent pas non plus en littérature, tels que le Lamiel de Stendhal/Jacques Laurent ou les nombreuses tentatives pour achever le 
Mystère d'Edwin Drood de Dickens, parfois à plusieurs mains (Fruttero et Lucentini) ${ }^{35}$. Il y a d'ailleurs bien d'autres raisons, moins sinistres, qui motivent de telles collaborations successives dans les arts à phase unique. La forme très particulière de contribution à plusieurs mains qu'est la traduction se déploie également en termes de successivité : le traducteur "collabore " avec l'auteur pour créer un cuvre commune, mais c'est par l'intermédiaire d'un texte déjà achevé (parfois depuis des siècles). Il y a donc deux phases distinctes, même si elles n'ont pas exactement la même nature que celles décrites par Goodman. Quant au cinéma, l'organisation classique du travail à Hollywood voulait que les scénaristes interviennent les uns après les autres sur un scénario, pour l'améliorer ou le transformer, avant qu'il ne soit transmis au metteur en scène pour exécution ${ }^{36}$.

En allant plus loin, on s'aperçoit toutefois que l'opposition entre successivité et simultanéité est plus superficielle qu'il n'y paraît. Il y a toujours de la successivité dans la cocréation simultanée. Les deux journalistes ne tapent pas au même moment sur le clavier et, pour se comprendre, ne parlent pas véritablement en même temps : chacune réagit à ce que l'autre vient d'avancer. Même l'extraordinaire osmose entre Aragon et Breton qui transparait dans les documents inédits publiés ici par Luc Vigier se traduit par l'enchaînement des écritures en cours de phrase et non par leur superposition. De même l'improvisation théâtrale peut généralement s'analyser comme un échange de répliques et l'improvisation musicale comme un perpétuel jeu d'ajustements et de rebonds sur les propositions des partenaires en interaction - ainsi que sur le canevas, protocole ou standard potentiellement à l' origine de leur improvisation. Dans tous ces cas, la simultanéité est en fait une alternance - alors que les collaborations successives ne laissent pas toujours la place à l'alternance, ne serait-ce que pour des raisons chronologiques.

Pour qu' il y ait collaboration au sens propre, il faut qu'il y ait impact du travail de chacun sur le travail de l'autre, et la véritable distinction porte sans doute sur le degré de réciprocité de cette action. À côté du modèle hollywoodien des scénaristes travaillant successivement à la révision d'un scénario avant qu'il ne parvienne au metteur en scène qui le tournera, il y a le modèle d'Aurenche, Bost et Autant-Lara, ou celui de Truffaut et Gruault, qui peuvent échanger et interagir à tous les stades d'écriture, depuis la première idée jusqu'au moment du tournage. Le metteur en scène se mêle étroitement de l'écriture et redigère, selon l'expression de Gruault, le langage du scénariste, qui doit de son côté fournir des idées de mise en scène, suggérer des mouvements de caméra et des jeux d'acteur.

35. Récemment, Friedemann Sallis a également posé la question du statut des musicologues qui complètent les œuvres inachevées des grands compositeurs (Music Sketches, Cambridge, Cambridge University Press, 2015 , chap. X). Quand peut-on dire qu'ils ont franchi la frontière entre rigueur scientifique (allant jusqu'à la reconstruction sonore de leur objet sur la base matérielle des manuscrits et documents de travail) et contribution créatrice en différé ? Et si le travail de Jacques Laurent rédigeant sa version de Lamiel doit être considéré comme une écriture collaborative, qu'en est-il du travail de Serge Linkès (Stendhal, Lamiel, dans CEuvres romanesques complètes, $t$. III, Paris, Gallimard, coll. «Bibliothèque de la Pléiade », 2014), remettant de l'ordre dans le manuscrit disparate laissé par Stendhal, afin d'en produire une version enfin convaincante pour le lecteur ? Son travail ne se mêle-t-il pas étroitement à celui de l'auteur ? Si c'est le cas, faut-il étendre la notion de collaboration au travail de tout éditeur scientifique mettant en valeur un texte canonique?

36. Stillinger (op. cit., p. 177-178) reproduit une terrible description de la sédimentation des écritures dans un script hollywoodien et du jeu stratégique auquel se livrent les scénaristes de manière à être le dernier intervenant sur le texte avant qu'il ne soit filmé et ainsi être celui qui sera «crédité » dans le générique, repoussant ses multiples prédécesseurs dans l'anonymat. 
Même dans le cas de la traduction, qui semblait devoir s'analyser en termes de successivité pure, il peut y avoir alternance et interaction, dès lors que l'auteur est vivant, sous forme de coopération constructive ou d'affrontement violent ${ }^{37}$ - quand le traducteur sollicite des éclaircissements de l'auteur, ou quand celui-ci exige et exerce un droit de regard sur la traduction. Cette interaction est toutefois limitée à la phase seconde, celle de la traduction du texte. Il en va différemment en musique quand l'interprète, sollicitant le compositeur par une commande ou sollicité par lui, prend une part active à la genèse de l' 'euvre qu' il sera ensuite appelé à jouer 38 : il n' est plus «interprète » au sens de celui qui vient s'approprier et exprimer le sens d'un texte déjà là, mais joue un rôle de cocréateur à la compétence spécialisée (en l'occurrence relative à la technique de son instrument). Enfin et par ailleurs, il n'est pas rare que les auteurs de théâtre remanient leurs pièces avant même la première représentation sous l'influence des metteurs en scène ou des comédiens vedettes.

En démontrant la nécessité d'une distinction entre genèse du texte et genèse de l'œuvre, l'article d'Anne Réach-Ngô tend à suggérer que la littérature pourrait, elle aussi, être rangée parmi les arts à plusieurs temps et donc intrinsèquement collaboratifs : au-delà de la phase d'invention du texte à laquelle, en général, s'intéresse exclusivement la critique génétique, il faut prendre en compte la mise en livre de ce texte et sa diffusion à un public. À nos yeux, le travail de l'éditeur produisant un livre à partir d'un texte, comparé à celui de l'architecte construisant un édifice sur la base de plans détaillés, du musicien jouant une partition ou du comédien interprétant une pièce, peut paraittre négligeable et tout à fait mécanique ${ }^{39}$. Mais les pratiques de la Renaissance, leur richesse, leur liberté et leur diversité, nous obligent à changer notre regard. Ce que nous considérons comme un même texte peut se manifester sous des formes si différentes du point de vue typographique, iconographique et plastique, être intégré à des appareils péritextuels si complexes qu'il en est profondément métamorphosé. Anne Réach-Ngô considère qu'il ne s'agit pas seulement d'interprétations différentes de la même æuvre, comme c'est le cas en musique, mais bien d'œuvres différentes. Quoi qu'il en soit, on constate ici encore des processus d'empiétements d'une phase sur l'autre : non seulement l'écrivain peut avoir son mot à dire sur la mise en livre de son texte (ce qui est conforme à nos habitudes modernes), mais il peut y avoir effet en retour de l'édition (dans sa matérialité d'objet comme dans sa diffusion) sur les versions ultérieures du texte 40 .

Cette problématique du temps de la collaboration est en fait beaucoup moins nouvelle pour la critique génétique qu'on ne pourrait le penser. On est confronté à des questions semblables quand on s'intéresse à Claudel remaniant en 1948 le Partage de midi écrit en 1905 et ajustant des positions esthétiques et éthiques fortement divergentes ${ }^{41}$, ou à

37. Voir sur ce point Olga Anokhina, «Traduction et réécriture chez Vladimir Nabokov : genèse d'une œuvre en trois langues », Genesis, n 38, « Traduire », 2014, p. 111-127.

38. C'est une collaboration de ce type que Nicolas Donin met en évidence entre le compositeur Luciano Berio et le hautboïste Heinz Holliger dans le présent numéro.

39. Voir Pierre-Marc de Biasi, « Pour une génétique de l'architecture », art. cité, p. 26.

40. Voir aussi le récent ouvrage de Roger Chartier, La Main de l' auteur et l' esprit de l' imprimeur, Paris, Gallimard, 2015.

41. Pour une vue d'ensemble de ce cas complexe, qui est en fait collaboratif à plus d'un titre, voir Almuth Grésillon, La Mise en æuvre. Itinéraires génétiques, Paris, CNRS Éditions, 2008, p. 260-264. 
Wordsworth produisant pendant un demi-siècle de multiples versions de son Prelude, esthétiquement diverses et politiquement contradictoires ${ }^{42}$. Il est clair que ces auvres, dissemblables bien qu' homonymes, n'ont pas vraiment le même auteur, ou plutôt qu'elles sont le fruit d'une collaboration étroite entre des versions successives de l'auteur ( "Claudel vieux » travaillant sur la base de ce que lui fournit «Claudel jeune »; "Wordsworth conservateur » adaptant les strophes de "Wordsworth révolutionnaire »). L' ouvre est alors littéralement un objet transitionnel, un truchement permettant la coopération de différentes incarnations temporelles de l'auteur.

Ces cas de disjonctions extrêmes sont en fait généralisables jusqu'au niveau le plus élémentaire : l'écrivain qui rature un mot et le remplace n'est pas exactement le même que celui qui l'a inscrit, même dans le cas d'une correction intervenant presque instantanément. Si celui qui relit avait exactement la même perspective que celui qui a écrit, il n'y aurait pas de ratures, pas de corrections, pas de nouvelles versions. C'est l'intervalle qui s'ouvre entre les deux positions qui crée l'espace dans lequel peut opérer la critique génétique 43 .

Le généticien a l'occasion de vérifier à longueur de manuscrits que l'œuvre, comme l'affirme Valéry, est une "fabrication à laquelle on ne pourrait pas faire correspondre un auteur agissant d'un seul mouvement. Elle est le fruit d'une collaboration d'états très divers, d'incidents inattendus ; une sorte de combinaison de points de vue originairement indépendants les uns des autres 44 » et «celui qui l'ê̂t pu faire d'un seul trait, sans déviations, sans interventions, n'a jamais existé45 ». Il est significatif que Valéry utilise ici, au sens propre ou au sens figuré, le mot de "collaboration ». Il indique bien que ce qui est à l' ceuvre dans la genèse, ce n'est ni la figure unificatrice de "l'auteur implicite46" engendré par l'œuvre achevée pour occuper la place du sujet de l'énonciation, ni l'entité juridique qui signe le texte et l'autorise, mais une multiplicité d'instances, temporellement disjointes, qui doivent composer l'une avec l'autre. Le travail de la critique génétique est précisément d'identifier ces instances multiples, de dé-niveler le texte de l'œuvre, d'y révéler la sédimentation des volontés diverses. En ce sens, la problématique de la création pluriauctoriale ne fait que rendre visible, incarnée dans des "mains " différenciées, un feuilleté polyphonique présent de façon parfois moins saisissable dans toute genèse, même la plus solitaire.

\section{Que reste-t-il de nos auteurs?}

Toutes ces extensions fonctionnelles et chronologiques soulèvent évidemment la question des limites du concept de collaboration. Jusqu' où peut-on aller, ou plutôt jusqu' où est-il utile d'aller dans l'inclusion des contributions créatrices au sein du projet génétique?

42. Voir Stillinger, op. cit., chap. IV.

43. Sur ce point, voir Daniel Ferrer, Logiques du brouillon. Modèles pour une critique génétique, Paris, Éditions du Seuil, coll. « Poétique », 2011.

44. Frédéric Lefèvre, Entretiens avec Paul Valéry (Paris, Le Livre, 1926), p. 107-108. C'est Valéry qui souligne. Cité par Judith Robinson-Valéry dans «Valéry précurseur de la génétique », Genesis, n 5, 1994, p. 97. 45. Vues (Paris, La Table ronde, 1948), cité dans «Valéry précurseur de la génétique » art. cité, p. 98. 46. Wayne C. Booth, The Rhetoric of Fiction, Chicago, University of Chicago Press, 1961. 
Howard Becker ouvre son célèbre livre sur les "mondes de l'art » avec une citation de Trollope, où celui-ci affirme que son auvre doit beaucoup, en tout cas plus qu'à quiconque, au domestique qui lui apportait son café tous les jours à cinq heures, lui permettant de se mettre au travail de grand matin ${ }^{47}$. Doit-on considérer cet homme comme un collaborateur de Trollope? Sans donner de réponse simpliste à cette question-là, Becker a plaidé en tout cas pour une sociologie compréhensive de l'ensemble des interventions, institutions, conventions, interactions qui font exister un monde de l'art et rendent possible (c'est-à-dire aussi performent activement) de nouvelles réalisations artistiques. Où s'arrêter ? Becker remarque que les génériques de cinéma, malgré leur longueur toujours croissante, ne sauraient rendre compte de tous les intervenants dans un film de quelque complexité. Où finit la liste des personnes dont la contribution est irremplaçable (c'est-à-dire sans lesquels le film serait autre, voire ne serait pas du tout) et où commence celle des contributions dont n'importe qui aurait pu s'acquitter aussi bien? Si on essaye d'être exhaustif, l'enchaînement des causes ouvre la voie à une régression infinie. Faut-il "créditer " le mécanicien qui a construit le métro qui a conduit les machinistes au studio? Les parents qui ont engendré les acteurs?

A contrario, qu'en est-il de ce qu'on pourrait appeler les anti-collaborateurs - ceux qui mettent des bâtons dans les roues de la création et qui peuvent donc avoir un effet considérable sur l' œuvre dès le cours même de sa genèse? On songe d'abord aux censeurs officiels, dont l'impact sur la genèse a été principalement étudié (et pour cause) par les textologues russes 48 . Faut-il ranger dans cette même catégorie les éditeurs qui pré-censurent, les amis bien intentionnés qui suggèrent des arrangements ? La frontière est floue, comme dans le cas, étudié ici même par Pierre-Michel Menger, des disciples de Bruckner qui, à son insu, introduisent dans ses symphonies des adaptations qui sont destinées non pas à éviter une censure politique qu'elles n'encourraient pas, mais à les rendre plus acceptables. La postérité a vu dans ces interventions l'émergence d'une autorité concurrente mais in fine illégitime, quand elle n'a pas simplement crié au vandalisme. Le ténor qui a obligé Mozart à écrire "Dalla sua pace », le très célèbre air de Don Giovanni, parce qu'il était incapable de chanter "Il mio tesoro », le morceau de bravoure prévu pour le rôle de Don Ottavio, doit-il être considéré comme un collaborateur ou comme un anti-collaborateur?

Par-delà les restrictions pratiques sur lesquelles on peut s'accorder, et que nous avons suggérées au fil des pages précédentes (valoriser les contributions irremplaçables, pleinement interactives, donnant lieu à reconnaissance réciproque - sinon à approbation - entre les instances auctoriales), il est clair que la réponse à la question de la délimitation des contributions créatrices est d'abord fonction des présupposés (implicites ou explicites, théorie indigène ou théorie exogène) de celui qui la pose - et, en particulier, de ses conceptions de $l$ " «auteur » et $d u$ "processus de création ». Ainsi, le garçon qui amène son café matinal à Trollope sera-t-il probablement exclu des contributeurs au processus créateur dans une perspective génétique, mais pourra l'être au sein d'autres épistémologies de l'étude de la création artistique. Néanmoins ces deux options supposent l'une comme l'autre d'avoir d'abord bien compris l'organisation de l'activité créatrice concernée, avec les hiérarchies

47. Howard S. Becker, Art Worlds, Berkeley, University of California Press, 1984, p. 1.

48. Voir Andrei Mikhailov et Daniel Ferrer (éd.). La Textologie russe : anthologie, Paris, CNRS Éditions, 2007 et Catherine Viollet et Claire Bustarret (dir.), Genèse, censure, autocensure, op. cit. 
qu'elle implique. Patrice Chéreau n'aurait pas admis l'idée d'une contribution créatrice de ses machinistes, et pourtant l'article de Marie-Madeleine Mervant-Roux révèle le soin avec lequel étaient choisis individuellement les principaux membres du "pôle technique " sur lequel s'appuyaient les mises en scène de Chéreau et toute l'inventivité dont chacun devait faire preuve. Chaque domaine artistique et chaque processus créateur particulier ont leurs modalités d'allocation des différentes fonctions d" "auteurs » et de " contributeurs » impliqués. La figure de l'auteur est une notion heuristique, non indispensable, puisque des civilisations, des époques et des genres entiers ont pu en faire l'économie, mais bien utile dans un cas comme le cinéma ${ }^{49}$ pour assigner à un référent stable des paquets de décisions et d'intentions, et plus ou moins naturel dans d'autres cas de figure.

Faire apparaître ainsi la multiplicité des contributions à l'œuvre et de leurs implications artistiques (plus ou moins profondes, plus ou moins volontaires et plus ou moins consenties) ne mène pas nécessairement à la mort de l'auteur par destitution. On le verra tout au long des pages qui suivent, Chéreau, Berio ou Bruckner furent absolument "auteurs » non seulement dans leur propre vision mais aussi dans celle qu'en avaient leurs collaborateurs, même les plus étroitement associés à des décisions ontologiquement cruciales pour l'ouvre finale. Il s'agit donc plutôt, dans le prolongement du travail pionnier de Stillinger, de nous défaire de nos préjugés sur la valeur des interventions sur l' euvre par d'autres que l'auteur nominal, de distinguer autorité et auctorialité (tant du point de vue des acteurs que de celui du chercheur, entre lesquels il convient également d'opérer un net distinguo) et de se demander ce que la reconnaissance des collaborations prolongées comme des interventions ponctuelles fait à la théorie génétique. C'est d'autant plus urgent qu'avec Internet un fabuleux laboratoire d'écriture collaborative est maintenant ouvert sous nos yeux, offrant à nos analyses, en temps réel, une masse de données telle qu'il n'en a jamais existé.

Nicolas Donin et Daniel Ferrer

49. Que cet auteur soit, suivant les cas, le metteur en scène, comme le voulait Truffaut, le producteur, le scénariste, voire l'acteur, ou le directeur de la photographie. Voir Jean-Loup Bourget et Daniel Ferrer, « Genèses cinématographiques », Genesis, n²8, « Cinéma », 2007.

Nicolas Donin est musicologue à l'Institut de recherche et de coordination acoustique/musique, où il dirige l'équipe Analyse des pratiques musicales (Ircam-CNRS-UPMC). Il est l'auteur d'études sur les processus de création musicale, l'histoire des pratiques d'écoute et d'analyse musicale depuis la fin du XIXe siècle, et l'analyse musicale d'interprétation, publiées à la fois dans le champ musicologique et dans celui des sciences humaines. Il est coauteur de courts-métrages, de DVD-Roms et de publications en ligne issus de ces recherches.

Nicolas.Donin@ircam.fr

DANiel Ferrer est chercheur à l'ITEM (ENS-CNRS). Il a écrit des livres et des articles sur Joyce (plus de soixante-dix), Woolf, Faulkner, Poe, Stendhal, Flaubert, Zola, Barthes, Cixous, sur la peinture, les humanités numériques, la génétique cinématographique et la théorie de la critique génétique. Ouvrages récents : Logiques du brouillon. Modèles pour une critique génétique (Seuil, coll. « Poétique », 2011) ; Renascent Joyce (Gainesville, University Press of Florida, 2013) et l'édition de Brouillons d'un baiser : premiers pas vers Finnegans Wake (Gallimard, coll. « Du monde entier », 2014).

daniel.ferrer@ens.fr 